\title{
L. G. Kovács and linear groups
}

\author{
A. S. Detinko and D. L. Flannery
}

Dedicated to the memory of Laci Kovács

\begin{abstract}
We survey the legacy of L. G. Kovács in linear group theory, with a particular focus on classification questions.
\end{abstract}

Classifying linear groups is an old problem. For given degree $n>1$, field $\mathbb{F}$, and group type, the task is to list irredundantly all subgroups of $\mathrm{GL}(n, \mathbb{F})$ of that type up to $\mathrm{GL}(n, \mathbb{F})$-conjugacy. If possible, each conjugacy class representative should be specified by a generating set of matrices. Examples of the group type are: finite, soluble, nilpotent, quasi-simple, maximal in its class (provided that each relevant subgroup of $\operatorname{GL}(n, \mathbb{F})$ is contained in a maximal), irreducible, primitive, monomial, generated by matrices with special properties (e.g., pseudoreflections), and so on.

To narrow the scope and thereby have a reasonable hope of solving a linear group classification problem, we impose extra conditions, such as the following.

- Characteristic: zero $(\mathbb{F}=\mathbb{C}$, the complex field, or $\mathbb{F}=\mathbb{Q}$, the rationals, are typical instances); positive (mostly finite $\mathbb{F}$ ).

- Degree: 'small', or otherwise restricted, according to the prime factorisation of $n$.

Furthermore, although $\mathrm{GL}(n, \mathbb{F})$-conjugacy is a natural classification criterion, we might ask for isomorphism class representatives instead. We may even limit ourselves to classifying subgroups of $\mathrm{SL}(n, \mathbb{F})$ or $\operatorname{PSL}(n, \mathbb{F})$.

Laci Kovács had an abiding interest in linear group theory. He was one of the first to realise the suitability of computer algebra systems as an environment for 
linear group classification: using a computer to aid in the compilation of lists, and applying implemented lists to solve related algorithmic problems.

Much of Laci's research dealing with representation theory and permutation groups has strong intersections with linear group theory. Especially pertinent here are the asymptotic bounds that he proved for finite soluble and nilpotent groups. We mention a few of these results, to give context; the paper by G. R. Robinson in this volume contains more detail.

In [9], Laci and J. D. Dixon derived a bound in terms of $n$ and $\mathbb{F}$ on the number of generators of a finite nilpotent subgroup of $\operatorname{GL}(n, \mathbb{F})$, where $\mathbb{F}$ is a finite degree extension of its prime subfield. Then Laci, R. M. Bryant, and Robinson extended that result to any finite group generated by its soluble radical and generalised Fitting subgroup [3]. The case of soluble linear groups was crucial (note also the paper [26] with H.-S. Sim on the number of generators of an abstract finite soluble group). But perhaps the most striking achievement in this area is [25]. Laci and Robinson prove that a finite completely reducible linear group of degree $n$ over any field can be generated by $\left\lfloor\frac{3 n}{2}\right\rfloor$ elements. Further contributions to linear group theory include [24], which establishes complete reducibility of representations of the monoid of $n \times n$ matrices over a finite field.

Below we discuss a research programme founded by Laci in the 1990s, that targeted difficult linear group classification problems. We describe how the objectives of this programme were carried out with some of his students and postdoctoral researchers. Laci guided the development of techniques and formulated major strategies in this programme.

\section{Background}

We begin with a sketch of historical background. See [32, Chapter III] and [34, Chapter 3, §4] for comprehensive surveys.

Early interest in soluble linear groups over finite fields stemmed from their connection to soluble permutation groups. C. Jordan gave a method (which can be viewed as an archetypal group-theoretic algorithm) to construct such linear groups from those of smaller degree. Jordan's treatment is cumbersome and does not give a full classification up to conjugacy. For finite groups in characteristic zero, it essentially suffices to classify the irreducible ones, dispensing with small degrees first. Degrees 2 and 3 were investigated by Jordan and F. Klein, amongst others. H. F. Blichfeldt's wonderful book [2] covers finite complex linear groups of degree at most 4 .

It became customary to ignore imprimitive groups. The emphasis was rather on primitive subgroups of $\operatorname{SL}(n, \mathbb{C})$ or their images in $\operatorname{PSL}(n, \mathbb{C})$, and sometimes 
groups were determined only up to isomorphism. The standard justification for this is as follows. Let $\mathbb{F}$ be algebraically closed, and $Z$ be the scalar subgroup of $\operatorname{GL}(n, \mathbb{F})$. Given $G \leq \mathrm{GL}(n, \mathbb{F})$ we may define $H \leq \mathrm{SL}(n, \mathbb{F})$ such that $G Z=$ $H Z$. Suppose that $G$ is irreducible (resp., primitive). Then $H$ is irreducible (resp., primitive), $H$ and $G$ have isomorphic central quotients, and $H$ is finite precisely when $G / Z(G)$ is finite. An advantage of this reduction is that there are only finitely many conjugacy classes of finite primitive subgroups of $\mathrm{SL}(n, \mathbb{F})$. However, as W. Feit has pointed out [17], producing a classification in $\operatorname{GL}(n, \mathbb{C})$ from one in $\operatorname{SL}(n, \mathbb{C})$ or $\operatorname{PSL}(n, \mathbb{C})$ is not at all straightforward. $\oplus$

Classifying finite primitive (or quasiprimitive) subgroups of $\operatorname{SL}(n, \mathbb{C})$ gained popularity in the lead-up to the classification of finite simple groups. After Blichfeldt, authors including R. Brauer, Feit, W. C. Huffman, J. H. Lindsey II, and D. B. Wales gave accounts for $n \leq 10$ (see [15, pp. 76-78] and [16]).

Another wave of activity began in the late 1940s, as soluble linear groups were recognised to play a fundamental role in the theory of infinite soluble groups. D. A. Suprunenko and his students obtained various classifications of soluble linear and permutation groups. Usually just the maximal soluble subgroups of $\mathrm{GL}(n, \mathbb{F})$ are described (each soluble subgroup lies in a maximal). For example, [30, Theorem 6, p. 167] classifies the maximal irreducible soluble subgroups of $\operatorname{GL}(p, q)$ up to conjugacy, $p$ prime, with an explicit generating set stated for each conjugacy class representative (cf. [6]). Many of the classification results for soluble matrix and permutation groups by Suprunenko and his school are summed up in [31]. That book also contains a classification of the maximal primitive soluble subgroups of $\operatorname{Sym}(n)$, where $n \in\left\{p^{q}, p^{q^{2}}, p^{q r} \mid p, q, r\right.$ prime $\}$.

Other notable classifications are in prime or prime-square degree: minimal irreducible groups [33, p. 2986], and irreducible $p$-groups over an algebraically closed field (S. B. Conlon). Aside from this work, and in contrast to the insoluble case, exhaustive classifications of soluble linear groups were rarely attempteduntil the advent of the research school directed by Laci.

\section{The Kovács School}

Some time ago, Laci, J. Neubüser, and M. F. Newman proposed an algorithm to construct maximal subgroups of low index in a finitely presented group [28, pp. 24]. Their algorithm relies on having a list of the primitive subgroups $H$ of $\operatorname{Sym}(n)$ where $n$ is the subgroup index. If $H$ is soluble then $n=p^{m}$ for some $m$ and prime $p$, and listing these permutation groups is equivalent to classifying the irreducible

\footnotetext{
†'Don't fall into the trap I fell into, Blichfeldt only classified the groups in dimension 4 in $P G L(4, \mathbf{C})$. It is a long way to go to $G L(4, \mathbf{C})$.'
} 
soluble subgroups of $\mathrm{GL}(m, p)$ up to conjugacy. The need for such information motivated the PhD project of Laci's student Mark Short [28].

Short's overall approach is based on theory of maximal irreducible soluble subgroups of $\mathrm{GL}(n, \mathbb{F})$, as in [30, Chapter $\mathrm{V}]$ and with antecedents in work of Jordan. Chapters 3-5 of [28] furnish a classification of the irreducible soluble subgroups of $\mathrm{GL}(2, q)$ for odd $q$ (A. Hulpke later found that two conjugacy classes of monomial groups were missing). Other necessary results for $\mathrm{GL}(r, q), r$ an odd prime, and for primitive soluble subgroups of $\mathrm{GL}(4, q)$, are provided. The listing in [28, Chapter 7] of imprimitive groups of degree 4 is supplemented by a CAYLEY computation. Using the methods in his thesis, Short classified the irreducible soluble subgroups of $\mathrm{GL}(n, p)$ up to conjugacy for all $p^{n}<256$. He implemented this classification as a data library and made it publicly available.

A subgroup of $\mathrm{GL}(n, q)$ of order coprime to $q$ can be 'lifted' to an isomorphic copy in $\mathrm{GL}(n, \mathbb{C})$, and the lifting respects absolute irreducibility. Despite this link, classification problems over $\mathbb{C}$ have a different flavour to those over finite fields. One complicating factor is that a classification of finite subgroups of $\operatorname{GL}(n, \mathbb{C})$ might entail an infinite list (whereas there are only finitely many finite primitive subgroups of $\operatorname{SL}(n, \mathbb{C})$ up to conjugacy). For the lists discussed here, we may introduce a parametrisation on certain families of matrices, so that each listed group is designated by an integer string that corresponds to a generating set made up from the parametrised families. A model for this sort of listing scheme is exhibited in [4]. Conlon classifies the finite irreducible $p$-subgroups of $\operatorname{GL}(p, \mathbb{F})$, where $\mathbb{F}$ is a field not of characteristic $p$ with all $p$-power roots of unity. Such a group is conjugate to a subgroup $G$ of the full monomial matrix group $C_{p}<C_{p}$. The subgroup of diagonal matrices in $G$ has index $p$ and (to guarantee irreducibility) must be nonscalar. Conlon gave presentations for groups in his list, and proved that any two of them are conjugate if they are isomorphic.

Attacks on other monomial group classification problems have followed the same basic pattern as in [4]. Suppose that $G \leq \mathrm{GL}(n, \mathbb{C})$ is monomial. Let $\pi$ be the natural projection of $G$ into $\operatorname{Sym}(n)$ whose kernel $D$ is the subgroup of diagonal matrices (that is, $\pi$ sends non-zero matrix entries to 1 ). First, all candidates for $\pi(G)$ are written down; namely the transitive $T \leq \operatorname{Sym}(n)$. Then we solve (S), the $T$-submodule problem: find all $D$ normalised by $T$ such that if $\operatorname{ker} \pi=D$ then $G$ is irreducible. The next step is the extension problem (E) for each $T$ and its accompanying $T$-modules. Lastly, we solve the conjugacy problem $(\mathrm{C})$, showing that each $\mathrm{GL}(n, \mathbb{C})$-conjugacy class is represented exactly once in our final list.

After the success of [4], composite degrees present a new challenge. The second author, another student of Laci's, classified the finite irreducible linear $p$ groups of degree $p^{2}$ for $p=2$ [18]. We say a little bit about the methods used (some of which appear in [1, 28] too). The submodule lattice of a direct sum of 
modules may be assembled from isomorphisms between sections of the summands, via a well-known theorem due to Goursat and Remak. This is applied to solve (S). Second cohomology features in the solution of (E) and (C): $\left|H^{2}(T, D)\right|$ is an upper bound on the number of conjugacy classes of $G \leq \mathrm{GL}(4, \mathbb{C})$ such that $\pi(G)=T$ and ker $\pi=D$. Lyndon-Hochschild-Serre spectral sequences are used to calculate the requisite orders. For each $T$ and $D$, precisely $\left|H^{2}(T, D)\right|$ extensions $G$ of $D$ by $T$ in $\mathrm{GL}(4, \mathbb{C})$ are constructed. Any remaining conjugacy between these extensions is eliminated by ad hoc means. Having dealt with the 2-groups, Flannery went on to classify all finite irreducible monomial subgroups of $\operatorname{GL}(4, \mathbb{C})[19]$.

At this juncture it is appropriate to note a question in the province of the submodule listing problem (S), that arose out of an algorithm suggested by Conlon for decomposing group characters. Let $p$ be a prime, $V$ be the central quotient of $C_{p} \propto\left\langle\left(C_{p}\right)^{n}\right.$, and $N$ be any finite normal subgroup of $V$. Conlon conjectured that the centre $Z$ of $V / N$ has order at least $p^{n}$. Examples are known where $Z \cong$ $C_{p}^{n}, Z \cong C_{p^{n}}$, and $|Z|$ is much greater than $p^{n}$. When $n=1$ there is nothing to prove. Conlon verified his conjecture for $2 \leq n \leq 4$ by a combination of hand and machine calculations.

As far as we know, Conlon's conjecture is unresolved. Laci's student Zoltán Bácskai made progress towards an affirmative proof, but eventually changed his thesis topic to classifying finite irreducible monomial subgroups $G$ of $\operatorname{GL}(p, \mathbb{C})$. Insoluble groups now crop up: $\pi(G)=T \leq \operatorname{Sym}(p)$ is either 'compulsory' (i.e., soluble, $\operatorname{Alt}(p)$, or $\operatorname{Sym}(p)$ ); or 'sporadic', with just 11 values of $p$ less than 1000 giving such a transitive group. Bácskai obtained a complete classification for $p \leq$ 11, and for arbitrary prime degree $p$ when $T$ is compulsory. In particular, the solution of (S) for all $T$ occupies Chapters 3 and 4 and Section 7.1 of [1] (observe that a finite soluble monomial subgroup of $\operatorname{GL}(p, \mathbb{C})$ is irreducible if and only if its diagonal matrix subgroup is non-scalar). Bácskai's thesis, which should be in the literature, contains many valuable results on linear group classification.

An irreducible linear group of prime degree is either primitive or monomial. Dixon and A. E. Zalesskii classified finite primitive subgroups of $\mathrm{SL}(p, \mathbb{C})$, and insoluble finite monomial groups of prime degree over an algebraically closed field, in [11, 12, 13]. The paper [11] has a traditional aim-classifying primitive unimodular linear groups over $\mathbb{C}$-and makes critical use of the classification of finite simple groups.

A further milestone in the Kovács programme was supplied by Burkhard Höfling, who worked as a postdoctoral researcher with Laci. In a long and interesting paper [21], Höfling settles the case of imprimitive non-monomial finite irreducible groups over $\mathbb{C}$ in smallest degree. He begins by considering the general situation of $G \leq \mathrm{GL}(2 n, \mathbb{C})$ with an unrefinable imprimitivity system of size 2 . Either $G$ has just one system, or it has three ([21, Theorem 2.4] is a broader statement; 
its proof cites [23]). This yields an initial split in the classification. To construct all $G$, one needs to know all primitive groups of degree $n$. Thus, as part of his solution in degree 4, Höfling classified the finite primitive subgroups of $\operatorname{GL}(2, \mathbb{C})$ up to conjugacy. Each such group is contained in a central product of scalars with a primitive subgroup of $\mathrm{SL}(2, \mathbb{C})$, and the latter were classified previously [2, Chapter III]. Höfling's lists, together with [1, 19] and [2, Chapters V, VII] filled out to $\mathrm{GL}(n, \mathbb{C})$, would complete the Kovács programme in degrees less than 5 over $\mathbb{C}$. Degree 5 is surely achievable too, with [1,11] as a foundation.

Laci's student Hyo-Seob Sim wrote several papers on metacyclic linear groups. In [29], he examines the structure of metacyclic primitive subgroups of $\operatorname{GL}(n, q)$, with the intent to classify these groups when $n$ is an odd prime power (cf. the classification [8] of nilpotent primitive subgroups of $\mathrm{GL}(n, q)$ for all $n, q$; and the GAP procedure in [7] that returns the groups for input $n, q)$. Laci and Sim in [27] give a condition to decide whether two nilpotent metacyclic irreducible groups

$G, H \leq \operatorname{GL}(n, \mathbb{F})$ of odd order are conjugate. They isolate the subgroup $G^{\text {Aut } G}$ of $G$ whose elements are fixed under $\operatorname{Aut}(G)$, and show that (with caveats) the number of $\mathrm{GL}(n, \mathbb{F})$-conjugacy classes of subgroups $H$ of $\mathrm{GL}(n, \mathbb{F})$ isomorphic to $G$ is equal to the number of equivalence classes of faithful irreducible $\mathbb{F}$-representations of $G^{\mathrm{Aut} G}$.

\section{Related work}

Linear group classifications of the kind advocated by Laci are constantly in demand, and hence worth pursuing. The area is effectively still wide open. We review a sample of other classifications with a computational aspect that relate to Laci's concerns.

Dixon and B. Mortimer [10] listed all primitive permutation groups of degree less than 1000 with insoluble socle. Short originally aimed to match this range of degrees for soluble groups. B. Eick and Höfling [14] extended the degree bound far beyond that in [28]. Taking Aschbacher's categorisation of potentially maximal subgroups of $\mathrm{GL}(n, q)$ as a starting point, they developed an algorithm to classify the soluble irreducible subgroups of $\operatorname{GL}(n, p)$ for $p^{n} \leq 6560$. (Höfling later augmented the list, going up to permutation group degree 10000.) Their algorithm involves testing irreducibility and subgroup conjugacy in $\operatorname{GL}(n, q)$. The complexity of this algorithm grows with $n$ and $q$. More recently, H. J. Coutts, M. Quick, and C. Roney-Dougal [5] classified the insoluble irreducible subgroups of $\operatorname{GL}(n, p)$ for $p^{n}<4096$.

Building on [19], and at the instigation of Laci, Flannery gave methods to classify the irreducible monomial subgroups of $\mathrm{GL}(4, q)$. Subsequently Flannery 
and E. A. O'Brien [20] designed algorithms to list irreducible linear groups of small degree over finite fields, with analogous classifications over $\mathbb{C}$ as an ingredient. The input field size bounds the integer parameter strings that define generating sets. A key theorem in [20] asserts that if $\mathbb{F}$ is any extension of $\operatorname{GF}(q)$, and $n \geq 3$ or $q>3$, then a subgroup of $\mathrm{GL}(n, \mathbb{F})$ isomorphic to $\mathrm{SL}(n, q)$ is irreducible and conjugate to $\operatorname{SL}(n, q)$. The proof of this result is mainly due to Laci and uses [22]. Implementations of the algorithms of [20] are available in MAGMA. Their efficiency depends on field arithmetic (as do, e.g., the algorithms of [7, 8]), and they avoid testing irreducibility or conjugacy. The input field size is unrestricted except for a tiny number of exceptions. This type of implementation may be compared with data libraries such as [28], and with the approach of [14], which requires non-trivial computation in $\operatorname{GL}(n, q)$. Techniques similar to those in [20] could be applied at least up to degree 5 .

Classifying irreducible soluble linear groups over a finite field in other special degrees, such as the product of two primes, is feasible. Suprunenko's book [31] is yet to be exploited for this purpose. The resultant algorithms would be practical for large degrees and fields.

Finally, we note that progress in computational representation theory affords new avenues for classifying insoluble linear groups over finite fields and $\mathbb{C}$.

\section{Concluding remarks}

The second author remembers hours spent each week with Laci, seeing the solution of a problem through to its very end. Laci was extremely generous in sharing his expertise. The work that he inspired and nurtured in others forms an important part of his legacy. We owe him a lasting debt.

\section{Acknowledgment}

The authors were supported by the Irish Research Council (grant 'MatGpAlg') and Science Foundation Ireland (grant 11/RFP.1/MTH/3212). A. S. Detinko was further supported by a Marie Skłodowska-Curie Individual Fellowship grant H2020 MSCA-IF-2015, No. 704910 (EU Framework Programme for Research and Innovation).

\section{References}

[1] Z. Bácskai, Finite irreducible monomial groups of small prime degree. $\mathrm{PhD}$ thesis, Australian National University, 1999. 
[2] H. F. Blichfeldt, Finite collineation groups. University of Chicago Press, Chicago, 1917.

[3] R. M. Bryant, L. G. Kovács, and G. R. Robinson, Transitive permutation groups and irreducible linear groups, Quart. J. Math. Oxford Ser. (2) 46 (1995), no. 184, 385-407.

[4] S. B. Conlon, p-groups with an abelian maximal subgroup and cyclic center, J. Austral. Math. Soc. Ser. A 22 (1976), no. 2, 221-233.

[5] H. J. Coutts, M. Quick, and C. Roney-Dougal, The primitive permutation groups of degree less than 4096, Comm. Algebra 39 (2011), no. 10, 35263546 .

[6] A. S. Detinko, A new GAP group library for irreducible maximal solvable subgroups of prime degree classical groups, J. Math. Sci. (New York) 108 (2002), no. 6, 942-950.

[7] A. S. Detinko, B. Eick, and D. L. Flannery, Nilmat-Computing with nilpotent matrix groups.

[8] A. S. Detinko and D. L. Flannery, Classification of nilpotent primitive linear groups over finite fields, Glasg. Math. J. 46 (2004), no. 3, 585-594.

[9] J. D. Dixon and L. G. Kovács, Generating finite nilpotent irreducible linear groups, Quart. J. Math. Oxford Ser. (2) 44 (1993), no. 173, 1-15.

[10] J. D. Dixon and B. Mortimer, The primitive permutation groups of degree less than 1000, Math. Proc. Cambridge Philos. Soc. 103 (1988), no. 2, 213-238.

[11] J. D. Dixon and A. E. Zalesskii, Finite primitive linear groups of prime degree, J. London Math. Soc. (2) 57 (1998), no. 1, 126-134.

[12] J. D. Dixon and A. E. Zalesskii, Finite imprimitive linear groups of prime degree, J. Algebra 276 (2004), no. 1, 340-370.

[13] J. D. Dixon and A. E. Zalesskii, Corrigendum: "Finite primitive linear groups of prime degree" [J. London Math. Soc. (2) 57 (1998), no. 1, 126134], J. Lond. Math. Soc. (2) 77 (2008), no. 3, 808-812.

[14] B. Eick and B. Höfling, The solvable primitive permutation groups of degree at most 6560, LMS J. Comput. Math. 6 (2003), 29-39 (electronic). 
[15] W. Feit, The current situation in the theory of finite simple groups. Actes du Congrès International des Mathématiciens (Nice, 1970), Tome 1, pp. 55-93. Gauthier-Villars, Paris, 1971.

[16] W. Feit, On finite linear groups in dimension at most 10, Proceedings of the Conference on Finite Groups (Univ. Utah, Park City, Utah, 1975), pp. 397407. Academic Press, New York, 1976.

[17] W. Feit, personal communication (1997).

[18] D. L. Flannery, The finite irreducible linear 2-groups of degree 4. Mem. Amer. Math. Soc. 129 (1997), no. 613.

[19] D. L. Flannery, The finite irreducible monomial linear groups of degree 4, J. Algebra 218 (1999), no. 2, 436-469.

[20] D. L. Flannery and E. A. O'Brien, Linear groups of small degree over finite fields, Internat. J. Algebra Comput. 15 (2005), no. 3, 467-502.

[21] B. Höfling, Finite irreducible imprimitive nonmonomial complex linear groups of degree 4, J. Algebra 236 (2001), no. 2, 419-470.

[22] L. G. Kovács, Some representations of special linear groups, The Arcata Conference on Representations of Finite Groups (Arcata, Calif., 1986), 207-218, Proc. Sympos. Pure Math., 47, Part 2, Amer. Math. Soc., Providence, RI, 1987.

[23] L. G. Kovács, On tensor induction of group representations, J. Austral. Math. Soc. Ser. A 49 (1990), no. 3, 486-501.

[24] L. G. Kovács, Semigroup algebras of the full matrix semigroup over a finite field, Proc. Amer. Math. Soc. 116 (1992), no. 4, 911-919.

[25] L. G. Kovács and G. R. Robinson, Generating finite completely reducible linear groups, Proc. Amer. Math. Soc. 112 (1991), no. 2, 357-364.

[26] L. G. Kovács and H.-S. Sim, Generating finite soluble groups, Indag. Math. (N.S.) 2 (1991), no. 2, 229-232.

[27] L. G. Kovács and H.-S. Sim, Nilpotent metacyclic irreducible linear groups of odd order, Arch. Math. (Basel) 65 (1995), no. 4, 281-288.

[28] M. W. Short, The primitive soluble permutation groups of degree less than 256. Lecture Notes in Math., 1519, Springer-Verlag, Berlin, 1992. 
[29] H.-S. Sim, Metacyclic primitive linear groups, Comm. Algebra 22 (1994), no. $1,269-278$.

[30] D. A. Suprunenko, Matrix groups. Transl. Math. Monogr., vol. 45, American Mathematical Society, Providence, RI, 1976.

[31] D. A. Suprunenko, Permutation Groups. Navyka i Technika, Minsk, 1996 (in Russian).

[32] A. E. Zalesskii, Linear groups, Russian Math. Surveys 36 (1981), no. 5, 63128.

[33] A. E. Zalesskii, Linear groups. Translated from Itogi Nauki i Tekhniki, Seriya Algebra, Topologiya, Geometriya, Vol. 21, pp. 135-182, 1983.

[34] A. E. Zalesskii, Linear groups. Algebra, IV, Encyclopaedia Math. Sci., 37, pp. 97-196, Springer, Berlin, 1993. 